\title{
Objective measurement of gestalts: Quantifying grouping effect by tilt aftereffect
}

\author{
Ning Wei ${ }^{1,2} \cdot$ Tiangang Zhou ${ }^{1,2}$ (D) Lin Chen ${ }^{1,2}$
}

Published online: 26 October 2017

(C) Psychonomic Society, Inc. 2017

\begin{abstract}
The importance of holistic perception is described by Gestalt psychology and its principles. Gestalt psychologists have promoted a formal and testable framework for these principles. Quantitative measurements have been introduced from Gestalt psychology in order to complement traditional phenomenal descriptions. Here we demonstrated a new method of measuring grouping effects objectively and quantitatively, by means of tilt aftereffect (TAE) from visual adaptation. Experiment 1 validated the method by measuring grouping based on either proximity or color similarity. Experiments 2 and 3 verified that this paradigm is also effective with dimotif lattices in which different perceptual organizations compete. The novel TAE-based paradigm is an objective and effective method for studying perceptual organization, especially the relationship between different Gestalt principles.
\end{abstract}

Keywords Competing organization · Tilt aftereffect (TAE) Objective measurement

Perceptual grouping is essential for visual object recognition. It relies upon a set of grouping principles to organize elements within our visual field. Wertheimer first posed Gestalt laws of perceptual grouping by asking what stimulus factors influence the perceived grouping of discrete elements (Wertheimer, 1922, 1923). The principles of grouping that he elucidated

Tiangang Zhou

tgzhou@bcslab.ibp.ac.cn

1 State Key Laboratory of Brain and Cognitive Science, Institute of Biophysics, Chinese Academy of Sciences, Beijing, China

2 University of Chinese Academy of Sciences, Beijing, China included proximity, similarity, uniform density, good continuation, and common fate. Since then, some new principles of perceptual grouping have been proposed, including common region (Palmer, 1992), element connectedness (Palmer \& Rock, 1994), and synchrony (Alais, Blake, \& Lee, 1998; Blake \& Yang, 1997; Lee \& Blake, 1999). The phenomenological demonstrations were crucial for establishing the basic principles of organization. However, these studies commonly relied on descriptions of conscious experience in terms of the units that people naturally perceive, rather than the artificial ones imposed by standard scientific methods.

Modern vision scientists have elaborated some of these principles by studying them quantitatively and clarifying the conditions under which they operate (Wagemans et al., 2012). Oyama (1961) was the first to measure the strength of grouping by proximity. He recorded the amounts of time that participants reported seeing competing horizontal and vertical groupings, and used the time ratio of perceptual grouping as a function of distance ratio. Later, Kubovy and colleagues presented different kinds of dot lattices and asked participants to indicate the perceived orientations (Kubovy, Holcombe, \& Wagemans, 1998; Kubovy \& Wagemans, 1995). The frequencies of these perceived orientations over a large number of trials were used as estimates of the probabilities. This way of testing grouping has been used frequently in the recent literatures (Claessens \& Wagemans, 2005, 2008; Kubovy \& Van den Berg, 2008).

In addition to quantitative measurement, another typical challenge of studying perceptual organization is what rule governs when multiple grouping laws apply concurrently. To investigate this question, several studies were conducted using similar strategies (Hochberg \& McAlister, 1953; Kubovy \& Van den Berg, 2008; Oyama, Simizu, \& Tozawa, 1999; Quinlan \& Wilton, 1998; Rush, 1937). For the stimuli, the distance between dots in one orientation was held constant, 
and the luminance differences or distances between the adjacent elements were manipulated. Therefore, a series of grouping indifference curves were fitted in order to characterize the relation between the two grouping principles studied. More recently, Claessens and Wagemans (2008) presented a Bayesian model for contour detection based on proximity and collinearity grouping. Their method was based on simplified interactions between two gestalts that acted as independent variables in dot lattices.

Although quantitative measurements have been introduced in modern studies of competing grouping principles, these researches have tended to make use of alternative forced choices, producing data that do not necessarily reflect the characteristics of the stimulus itself. For example, when participants are forced to choose between indeterminate options, their answers may depend on conflicting cues from local features (e.g., dots with same color or dots with closer intervals) instead of on holistic perception of the gestalt. The perception of feature "similarity" itself and the perception of the Gestalt are confounded in the task. Participants' reports are likely controlled by top-down influence. As a result, such reports cannot truly reflect the perceptual organization process, and measurements are therefore biased. A famous example is the fragmented black-and-white image of a Dalmatian. If the participant is told that the image contains a dog, the percept can easily be identified. But without this information, the image may seem rather unstructured, and even incomprehensible. In a more relevant study, Beck and Palmer (2002) informed their participants about the percentages of trial types. Participants were then influenced by top-down processes, and subjective biases were induced. Kubovy also found that participants were more likely to apply the same grouping rule as they had for the preceding stimulus (Kubovy \& Wagemans, 1995). Therefore, a carefully designed task is needed to eliminate the effects of local features and top-down processing in the study of the Gestalt laws.

Palmer proposed an indirect quantification method called the repetition discrimination task. In a speeded discrimination task, participants were asked to report the shape of repeated elements in a line of otherwise alternating shapes (e.g., squares and circles). Then the difference between these two reaction times was used as a measure of the strength of the grouping effect (Palmer \& Beck, 2007). Unlike in the studies mentioned above, the participants' task was independent from perceptual grouping itself, so that subjective strategies had no influence on the grouping process.

In the present study, we proposed another indirect method to quantify competing grouping principles, in which two separate Gestalt principles operate at the same time in one stimulus. In adaptation studies, the paradigm of tilt aftereffect (TAE) relies upon the phenomenon in which perceived orientation of a test stimulus is altered after prolonged exposure to an oriented adaptor (Gibson \& Radner, 1937; He \& MacLeod,
2001). As an appearance-based psychophysical tool, this paradigm may effectively characterize the relationship between two Gestalt principles. We hypothesized that the grouping of a dot lattice would be perceived like oriented lines and lead to TAEs. In other words, TAE could be used to measure the grouping effect, while its magnitude might be modulated by strengths of different grouping effects.

In this article, we present three experiments demonstrating the validity of the TAE for studying perceptual grouping of a dot lattice. At the same time, we applied this new paradigm to test the relationship between proximity and color similarity. In Experiment 1 we validated this method using the classic Gestalt principles of proximity and color similarity. In Experiment 2, we extended the method to a dimotif lattice stimulus in which proximity and color similarity were arranged orthogonally. In Experiment 3, we modulated the grouping effects in dimotif lattices to verify the accuracy of this method.

\section{General method}

\section{Participants}

The participants were university undergraduates who received hourly pay for their participation. All were right-handed and reported normal or corrected-to-normal vision and no known neurological or visual disorders. They were all naïve as to the purpose of the experiment. The numbers of participants in Experiments 1, 2a, 2b, and 3 were eight, nine, five, and three, respectively.

\section{Experimental setup}

Stimuli The stimuli were displayed on a 22 -in. computer monitor (ViewSonic P220F) with a resolution of $1,024 \times$ 768 pixels $\left(1\right.$ pixel $=0.03^{\circ}$ at a viewing distance of $70 \mathrm{~cm}$ ). The experiment was programmed and run in MATLAB 2009a with the Psychophysics Toolbox extensions (Brainard, 1997; Pelli, 1997). Participants were seated in a room with dim ambient light, and their head position was stabilized using a chinand head-rest.

Design For all three experiments we used the same paradigm for detecting TAEs. Stimuli were displayed on a black background with a visual angle of $7^{\circ}$ (Fig. 1). Each block began with a 20 -s preadaptation period. In each trial, after a 5-s topping-up adaptation and a 0.2 -s blank interval, a test stimulus was presented for $0.2 \mathrm{~s}$, and participants were asked to make either a two-alternative forced choice (2-AFC; clockwise or counterclockwise $[\mathrm{CW} / \mathrm{CCW}]$ relative to vertical) or a four-alternative forced choice (4-AFC; $\mathrm{CW} / \mathrm{CCW}$ relative to horizontal or $\mathrm{CW} / \mathrm{CCW}$ relative to vertical) judgment as 


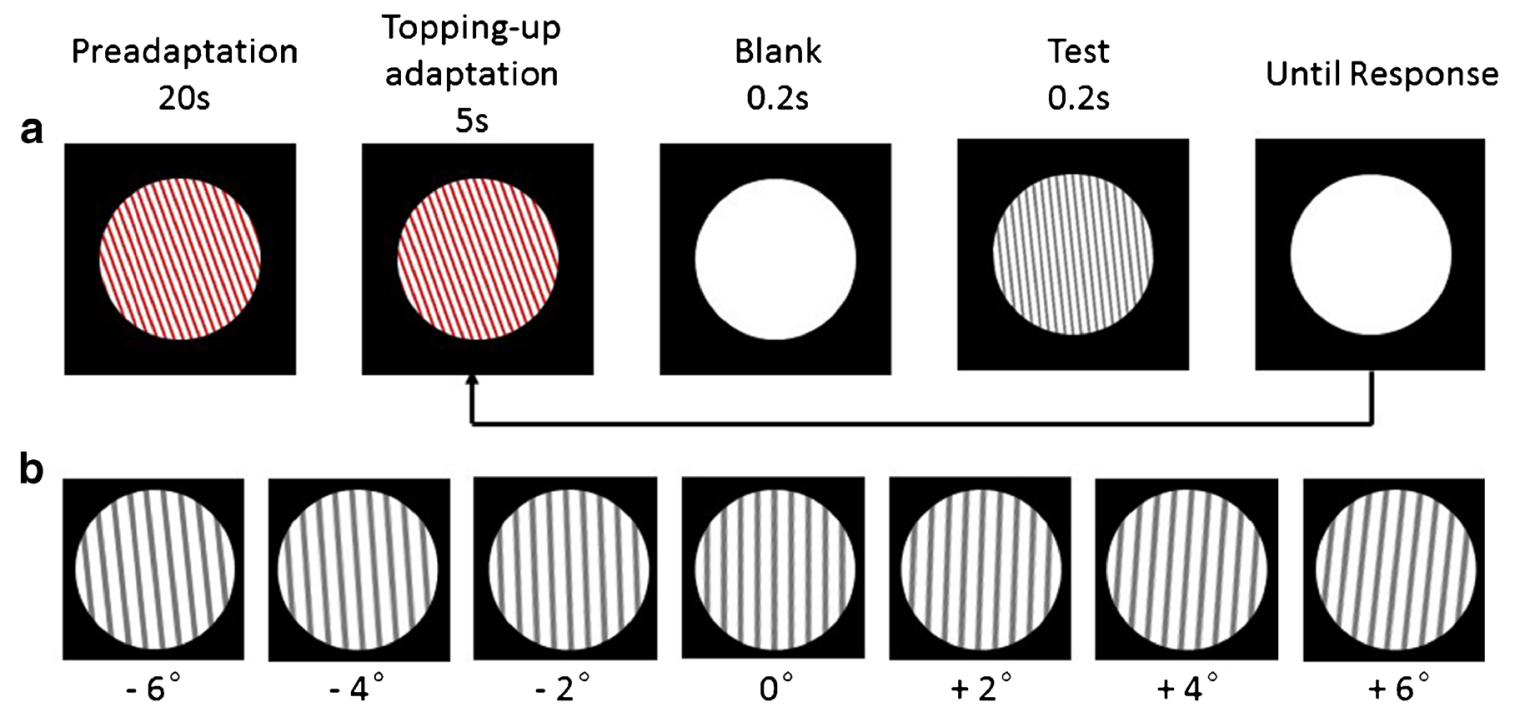

Fig. 1 (a) Schematic representation of our adaptation and test procedure. (b) Grating test stimuli for measuring tilt aftereffect

quickly as possible. During the periods of preadaptation and topping-up adaptation, the stimuli flickered at $2.5 \mathrm{~Hz}$. In accordance with previous studies (Gibson \& Radner, 1937), the orientations of the adaptation stimuli in our experiments were set to $\pm 20^{\circ}$ to reach the TAE peak. To minimize the effects of irrelevant factors on TAE, we used the same luminance and color contrast (Flanagan, Cavanagh, \& Favreau, 1990; Held \& Shattuck, 1971; Lovegrove \& Over, 1973) between the adaptors and test stimuli.

In all experiments, to control participants' attention during the adaptation phase, they were required to focus on the stimuli during the experimental period and to respond as quickly and accurately as possible. Before each experiment, participants were given oriented gratings to acquaint them with the key-press responses. They rested for approximately $3 \mathrm{~min}$ between blocks to minimize adaptation carryover from the previous block.

\section{Experiment 1}

In previous studies of TAE, tilted gratings were the typical stimuli used to produce adaptation of orientation (Gibson \& Radner, 1937; He \& MacLeod, 2001). In Experiment 1 we examined whether the orientation organized by the proximity or color similarity of dots could produce results similar to the TAEs observed by tilted gratings. Proximity and color similarity are the most basic, robust, and well-studied grouping principles (Brooks, 2015).

\section{Method}

Arrays of dots were shown on a white circular background (Fig. 2). In the control condition, the stimulus was a red grating (radius, $3.50^{\circ}$; spatial frequency, 2.86 cycles/deg; width of red stripe, $0.12^{\circ}$; width of white stripe, $0.23^{\circ}$; contrast, 6 $\left(\mathrm{L}_{\text {max }} / \mathrm{L}_{\text {min }}\right)$; orientation, $\left.\pm 20^{\circ}\right)$. In the other two conditions, the bars in the stimulus were replaced by dots (the diameter of the dots was $0.12^{\circ}$ ) based on proximity or color similarity principles. In the proximity condition, the distance between two red dots was $0.058^{\circ}$ along the dotted grating direction. In the color similarity condition, dots of the same color were placed in one direction, with spatial intervals identical to the gap between two adjacent rows.

The test stimuli were circular gray gratings (radius, $3.50^{\circ}$; contrast, $6\left(\mathrm{~L}_{\max } / \mathrm{L}_{\min }\right)$; spatial frequency, 2.86 cycles/deg; orientation, $0^{\circ}, \pm 2^{\circ}, \pm 4^{\circ}$, and $\pm 6^{\circ}$; shown in Fig. 1b). Their luminance was consistent with the corresponding adaptor during adaptation. One of the seven gratings was presented in the middle of the visual field as the test stimulus for measuring TAE. Participants were asked to indicate by key-pressing whether the test grating in each trial was tilted $\mathrm{CCW}$ or $\mathrm{CW}$ from the vertical.

Two adaptors of $\pm 20^{\circ}$ were presented alternately in the adaptation phase, for a total of eight blocks. Each of the seven test stimuli was presented six times in a block. The control, proximity, and color similarity conditions were tested randomly on separate days.

\section{Results}

The perceptual mislocalization of the adapting grating produced by the TAE was measured individually for each participant. Participant responses as a function of grating position were fitted with logistic functions using a least squares procedure. As is shown in Fig. 3a, the size of the TAE is equal to half the difference between the points of subjective equality (PSEs) in the two adaptation direction conditions (Kosovicheva et al., 2012), which is: 


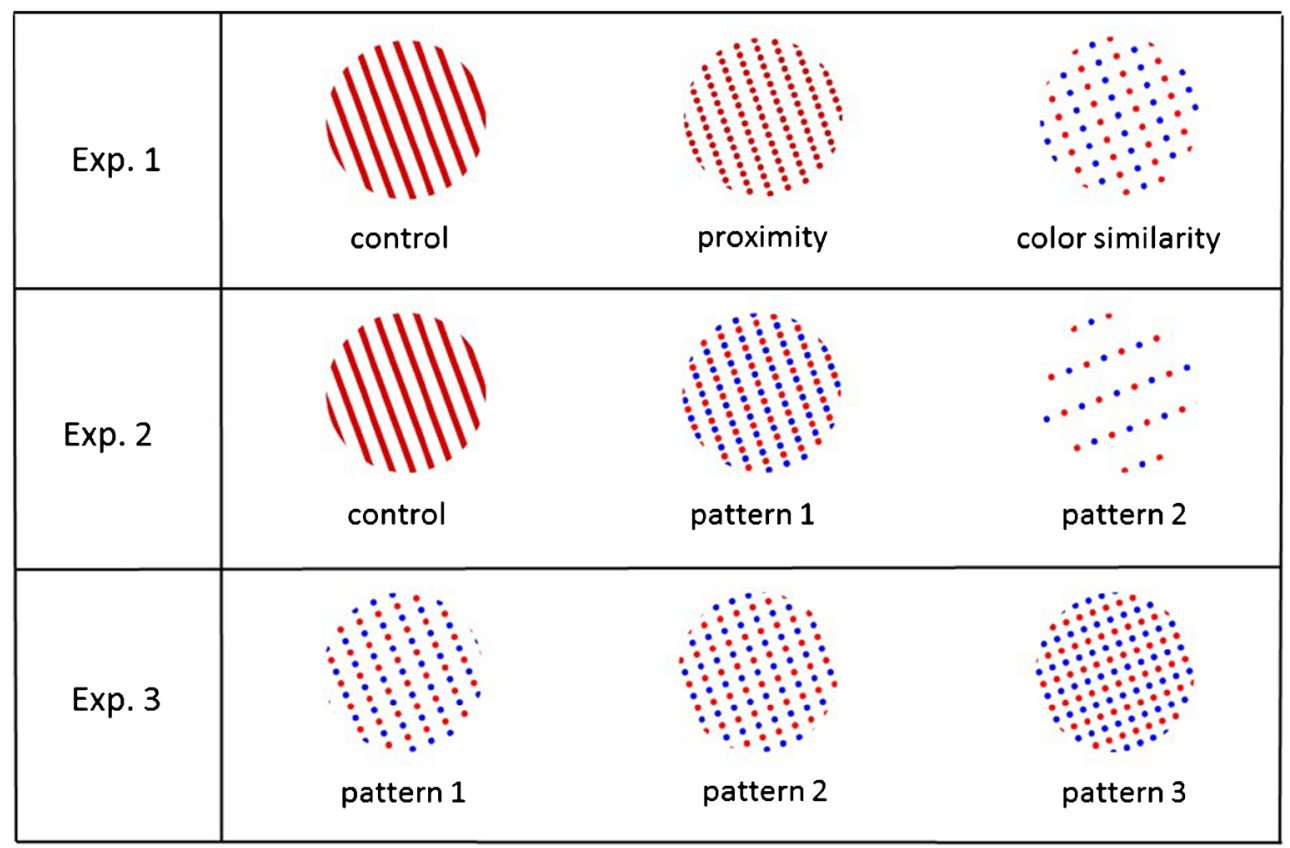

Fig. 2 Overview of the stimuli presented in the three experiments. In Experiment 1 we examined orientations formed by different grouping principles. Dimotif lattices were introduced in Experiment 2 in order to

$\mathrm{TAE}=\frac{P S E_{\text {adapt right }}-P S E_{\text {adapt left }}}{2}$.

Figure $3 \mathrm{~b}$ shows the TAEs obtained in the control (black bars), proximity (red bars), and color similarity (blue bars) conditions for the eight participants and the average across participants. The TAEs of all three conditions were significantly above zero (mean control condition: $M=2.64, S E=$ $0.22, p<.001$; proximity condition: $M=2.08, S E=0.17, p<$ .001 ; color similarity condition: $M=1.24, S E=0.15, p<$ $.001)$. The consistency among the three conditions was quite strong. Several findings were noteworthy. First, conditions of test competing organizations. The relative strengths of the groupings were adjusted in Experiment 3

both proximity and color similarity could produce significant TAEs. This is the predicted effect of proximity and color similarity grouping in this adaptation paradigm. In this way, participants easily perceived orientation information as in the control condition, so that a TAE could be observed in the results. This indicates that TAE is a plausible measurement of grouping effects. More importantly, as compared to color similarity, the proximity induced a significant larger TAE $[t(7)$ $=6.389, p<.001$, two-tailed paired $t$ tests]. We interpreted the difference in TAEs as measuring the strength of grouping in the display. A large TAE indicates that the organization is strong. a

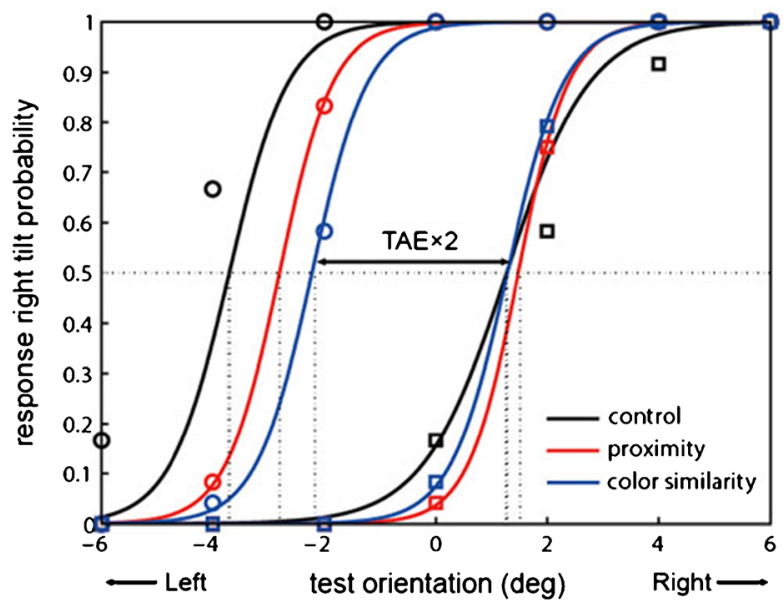

b

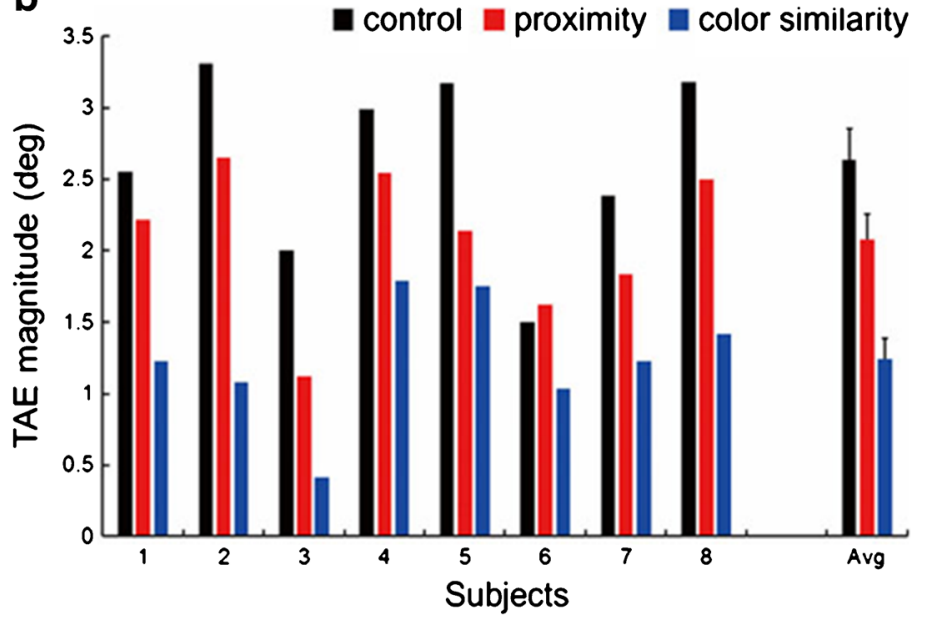

Fig. 3 (a) Example tilt aftereffect (TAE) measurement data from one participant. (b) Results for each of the eight participants, along with the average across participants. Error bars represent $1 S E$ 


\section{Experiment 2a}

In Experiment 1, we were able to obtain significant TAEs for both proximity and color similarity grouping effect conditions. We set out to compare the two Gestalt principles directly by presenting participants with patterns containing both proximity and color similarity at the same time.

In dimotif lattice stimuli, two kinds of elements are used to pit grouping by proximity against grouping by color similarity (Grünbaum \& Shephard, 1987); participants are able to perceive two orientations, based on either the proximity or color similarity Gestalt principle. We predicted that if we were to test participants using such a stimulus, we would obtain TAEs for both possible orientations, and the stronger grouping effect would cause a greater TAE magnitude. By comparing the values of the two TAEs, the relative strengths of the two grouping principles could thus be evaluated. If this proved true, TAE would be a quantitative method for evaluating the strengths of the competing principles.

\section{Method}

The adaptation stimuli in this experiment are shown in Fig. 2. The control condition was a red-striped grating with the same parameters as the control in Experiment 1. The condition containing dimotif lattice stimuli was the focus in this experiment. As is illustrated in Fig. 2, Pattern 1 (radius, $3.50^{\circ}$; spatial frequency, 2.86 cycles/ $\mathrm{deg}$; contrast, $6\left(\mathrm{~L}_{\max } / \mathrm{L}_{\min }\right)$; orientation, $\left.\pm 20^{\circ}\right)$ contained both proximity and color similarity grouping principles. Each string of dots in the direction of the proximity principle $(|p|)$ contained alternating instances of the two motifs (red and blue dots). All strings oriented in this direction had identical arrangements of the motifs. On the other hand, each string of dots oriented in the direction of the color similarity principle $(|\mathrm{c}|)$ consisted of only one of the motifs (e.g., red dots), whereas the adjacent, parallel string consisted of the other motif (e.g., blue dots). $|\mathrm{c}| /|\mathrm{p}|$ was thus equal to 2 in these dimotif lattices. On the basis of experimental work by Kubovy (Kubovy \& Van den Berg, 2008), the proximity principle of this stimulus is significantly stronger than the color similarity principle.

Participants viewed an adaptation patch of the same sort as in Experiment 1, except that the test patch following adaptation was either vertical $\left(0^{\circ} / 180^{\circ}\right)$ or horizontal $\left(90^{\circ} / 270^{\circ}\right)$. The participants were not aware of whether the test stimulus would be vertical or horizontal, and they were instructed to make a 4AFC. If the orientation of a test stimulus was close to that of the adaptation stimulus, the TAE of proximity grouping was tested. Otherwise, the TAE of the color similarity grouping was tested. This is shown in Fig. 4.

\section{Results}

The TAE magnitudes of the two orientations formed by the stripe patterns and dimotiff lattices are demonstrated in Fig. 5a. These data were analyzed using a repeated measures analysis of variance with two within-subjects variables: adaptor (stripe pattern, dimotif lattices) and test orientation (stimulus orientation, orthogonal orientation). The analysis revealed a significant main effect of test orientation $[F(1,8)=$ $\left.175.19, p<.001, \eta^{2}=.96\right]$ and an interaction between the two variables $\left[F(1,8)=74.09, p<.001, \eta^{2}=.90\right]$, yet the main effect of adaptor was not significant $\left[F(1,8)=3.98, p>.05, \eta^{2}\right.$ $=.33]$. Significant TAEs were produced by both proximity $(2.26 \pm 0.13)$ and color similarity $(1.17 \pm 0.11)$. As expected, proximity resulted in a larger TAE than did color similarity $[t(8)=7.91, p<.001$, two-tailed paired $t$ tests $]$. This was in agreement with the subjective reports of the participants.

The individual data from the stripe pattern and dimotif lattice conditions are summarized in Fig. 5b. The TAE of the orientation for the stripe pattern was compared against the TAE of the proximity orientation in the dimotif lattices, and the orthogonal orientation of the stripes was compared against the orientation for the color similarity principle. Little or no TAE was expected in the orthogonal orientation of the stripe pattern, because no obvious cue oriented the participants in that direction. The gray dots lying below the diagonal line confirm that the TAE of the color similarity orientation was larger than that of the orthogonal orientation of the stripe pattern $[t(8)=4.68, p=.002$, two-tailed paired $t$ tests $]$. We can interpreted that the color similarity principle in the dimotif lattices provided orientation information that helped participants produce a TAE in that direction. The black dots lying above the diagonal line indicate that the TAE from the proximity orientation was notably smaller than that of the orientation in the control condition $[t(8)=7.71, p<.001$, two-tailed paired $t$ tests]. One possible reason is that the strength of proximity grouping was a little weaker than the uniformconnectivity stripes, adhering to the results we obtained in Experiment 1. Another potential reason is that there was a conflict between the factor of proximity and the factor of similarity. This competition may have reduced the orientation strength expressed by the proximity grouping principle.

\section{Experiment 2b}

In Experiment 2a, the dots along the color similarity grouping orientation were so densely arranged in the orthogonal direction that the participants' judgments of color orientation may have been affected. To avoid this, we halved the density of the dots in Pattern 2. In this way, the frequency of dots in the color similarity grouping in Pattern 2 was the same as that in the 


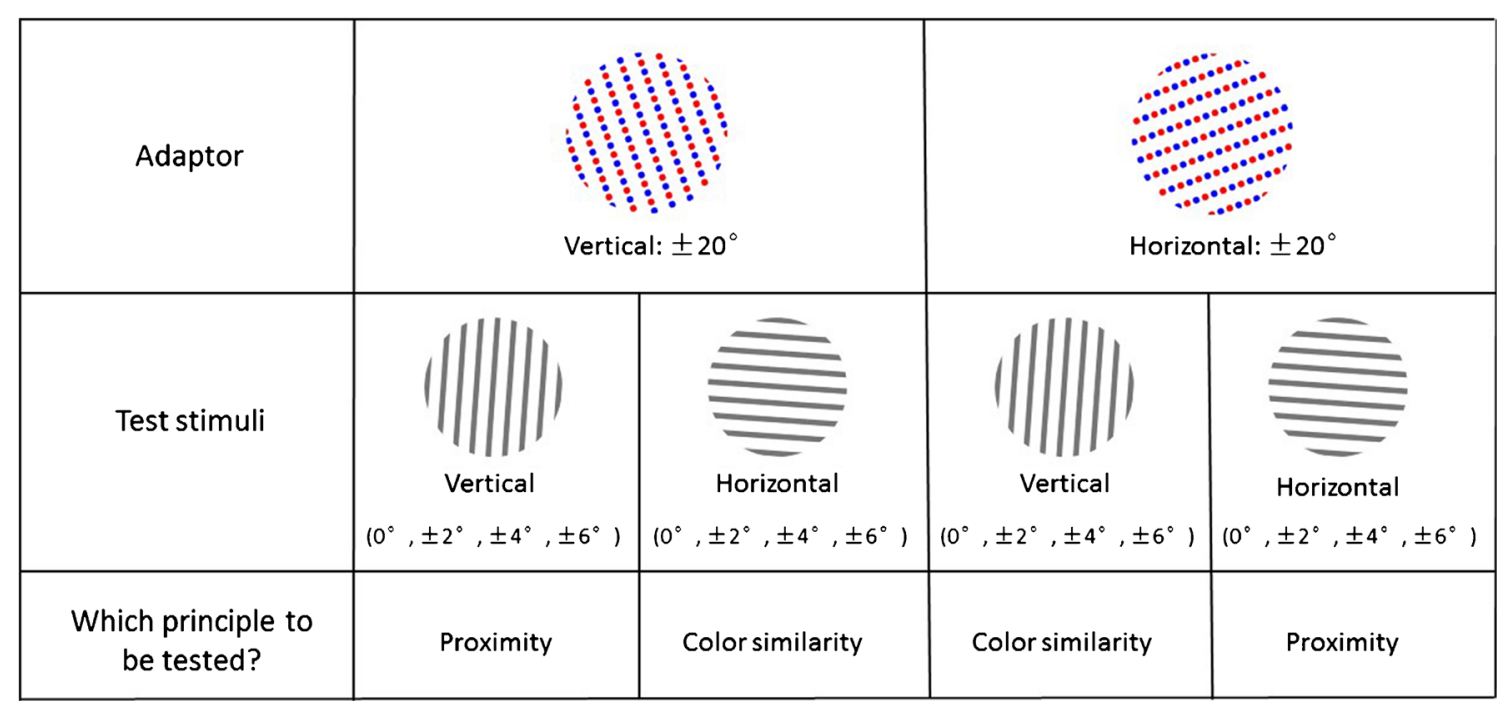

Fig. 4 Combinations of adaptor and test stimuli with the corresponding principles, which were tested in Experiment 2a

proximity grouping in Pattern 1 . We ran the experiment to see whether the density of dots had an effect on the results.

\section{Method}

The pattern of the dimotif lattices was replaced by Pattern 2, in which the density of the dots was reduced by half. All other parameters were consistent with those used in Experiment 2a. All of the participants in this experiment were those who had taken part in Experiment 2a.

\section{Results}

The results for the two patterns are compared in Fig. 6. No statistically significant difference was found between the two patterns. Therefore, the density of the dots had no effect on the results.

a
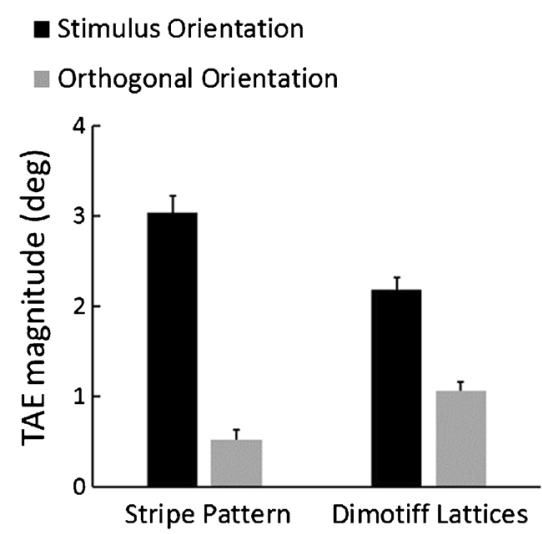

Fig. 5 (a) Comparing mean TAEs to our stripe pattern and dimotif lattice stimuli. Black bars refer to the orientation of the stripes and of the proximity principle in the dimotif lattices. Gray bars refer to the

\section{Experiment 3}

To further verify the effectiveness of the TAE method, we performed a third experiment using stimuli based on three different relative strengths of proximity and color similarity. We expected that when we compared participants' subjective ratings, the TAE method would give a quantitative representation of competitive interactions between the organization principles, especially in an ambiguous display.

\section{Method}

The procedure was the same as in Experiment 2, except for the stimuli. Here we used three different dimotif lattice stimuli, shown in Fig. 2. We kept the shortest distance between the two differently colored elements constant $\left(|c|=0.1^{\circ}\right)$ while adjusting the distance between dots of the same color $(|\mathrm{p}|)$.

b

$$
\begin{aligned}
& \text { - (proximity, stripe) } \\
& \text { - (color similarity, orthogobal to stripe) }
\end{aligned}
$$

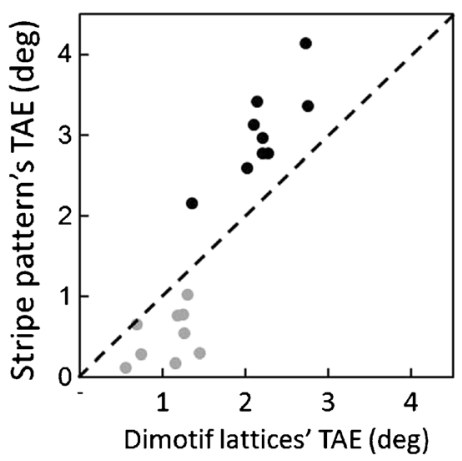

direction orthogonal from the stripes and to the orientation of the color similarity principle in the dimotif lattices. (b) Individual data from the two conditions 


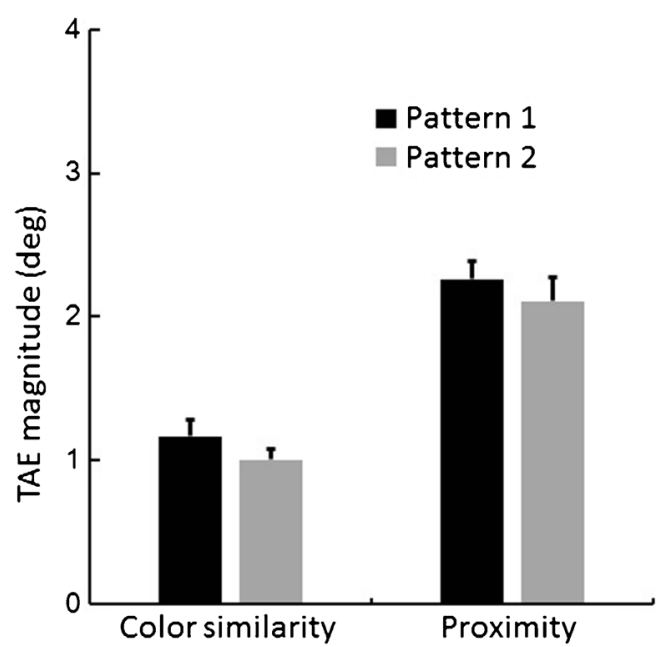

Fig. 6 Comparison of two different dot densities in Experiments $2 \mathrm{a}$ and $2 \mathrm{~b}$

As $|p|$ got smaller, the grouping effect of proximity became stronger. The proximity principle was dominant in Pattern 1, in which the ratio $|\mathrm{c} / / \mathrm{p}|$ was 1.125 . In Pattern 2 the dominant grouping was ambiguous, and the ratio $|\mathrm{c}| /|\mathrm{p}|$ was 1.375 . The two principles evoked a bistable orientation percept that fluctuated between vertical and horizontal groupings. The color similarity principle was dominant in Pattern 3, in which the ratio $|\mathrm{c}| /|\mathrm{p}|$ was 1.625 .

To compare these results with the corresponding subjective results, we added a subjective-report task after the adaptation task. The stimuli were the same as in the adaptation tasks. Each stimulus was presented in random order for 32 trials. Participants were required to report the dominant perceived orientation, as in Kubovy and Van den Berg's (2008) experiment.

\section{Results}

The results for the three participants are shown in Fig. 7. When the interval between dots of the same color was enlarged, the TAE of proximity grouping increased and the TAE of color similarity grouping decreased. Although we observed fluctuation in the TAE magnitudes, the relative predominances of the two grouping principles were consistent across all participants. In Pattern 1, the TAE of proximity grouping $(2.32 \pm 0.63)$ was significantly larger than the TAE of color similarity grouping $(0.86 \pm 0.20)$. In Pattern 3 , the principle of color similarity was dominant as compared to proximity, so that the TAE of color similarity $(2.02 \pm 0.29)$ was larger than that of proximity $(1.15 \pm 0.40)$. An interesting result in the competing groupings was exposed in the ambiguous Pattern 2: The TAE of the proximity principle was consistently larger than the TAE of the color similarity principle, indicating that all three participants perceived stronger proximity grouping than color similarity grouping, although their subjective reports were close to chance level.
There was strong consistency between the TAE results and the subjective reports. Although subjective-rating data are not necessarily a gold standard of participants' perceptions of grouping, they nevertheless were substantial enough to support the conclusion that the TAE provided a quantitative measurement of grouping strength that was consistent with subjective ratings. This consistency corroborates our expectations.

\section{Discussion}

According to the results of our experiments, the TAE method is a relatively novel and effective way to study grouping phenomena. Experiment 1 showed that both grouping by proximity and grouping by color similarity could produce TAEs. The results verified that the orientation formed by a grouping principle could be detected in this kind of adaptation paradigm, although different groupings differed in the strengths of their TAEs. Experiment 2 showed that when proximity and similarity groupings were applied concurrently in the same stimuli, TAE could be generated for both organized orientations. Experiment 3 further demonstrated this method's effectiveness with three different types of dimotif lattices. When we altered the spacing between the dots of the same color so that some dots were closer than others, and thus grouped together more strongly, the factor of relative distance influenced the strength of grouping.

This method is open to the objection that it might not measure grouping at all. However, the consistency of the TAE results with subjective ratings in Experiment 3 provides evidence that this method does indeed measure grouping. It is important that TAE is an indirect measurement of grouping. We never asked participants to report the grouping they perceived, and its organizational structure had no logical bearing on performing the task. The indirect and objective appearance of the measures makes it less susceptible to so-called demand characteristics of the experiment, in comparison to participants' subjective reports, which are affected by what participants believe the experimenter expects or wants.

Another question that deserves attention is the relationship between the two grouping principles. We interpreted the difference between the TAEs of the two orientations as a measurement of the relative grouping strengths in the display. A larger TAE indicated that the organization was stronger and formed a dominant orientation percept. Meanwhile, when one orientation was perceived, the other was nonetheless also present in the stimulus. How are these two grouping principles expressed? Do we switch from one perception to the other, or do they exist simultaneously? One reasonable explanation is that both of the two orientations could be represented in the stimulus percept. The grouping principle with higher strength would represent the stronger direction. This kind of grouping 


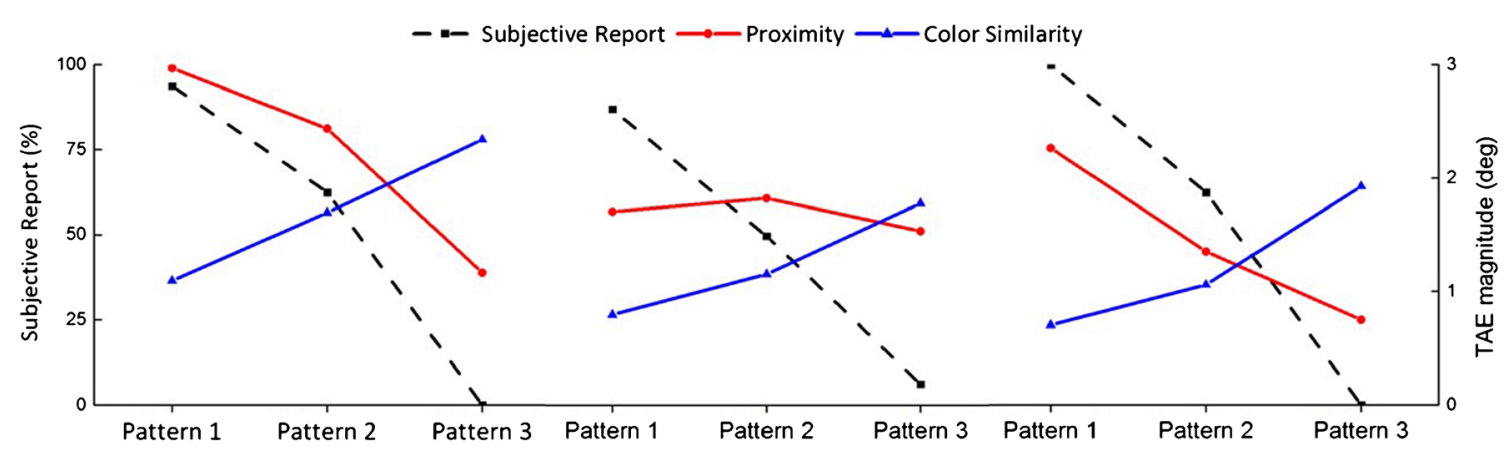

Fig. 7 Results from Experiment 3. Black dashes represent the rate at which subjective reports were consistent with the orientation formed by proximity. Red and blue lines show the TAE values for proximity and color similarity, respectively

tended to produce a higher TAE, as we demonstrated in the results of Experiment 3. When the orthogonal grouping became stronger, the TAE of the target grouping decreased due to competition.

Nevertheless, we need to declare that the TAE measure of competitive groupings will depend on the features of the stimuli. For example, when we changed the luminance difference between rows, the strengths of the groupings also changed, in that grouping by similarity was reduced and grouping by proximity was able to manifest itself. Thus, TAE results can only be used to give answers for specific stimuli, especially when their phenomenology is unclear.

In conclusion, this adaptation paradigm is an important method for the study of perceptual grouping. The indirect measurement of perceptual grouping has proved an objective way to evaluate the competition between different perceptual organizations. This method substantially reduces the likelihood that data might be contaminated by participants' subjective reports, which are affected by what they believe the experimenter expects. Another advantage is that this paradigm can be a useful method of probing the dominant grouping principle in any competition between organizations.

Author note This work was supported by the National Key Basic Research Program of China Grant 2015CB351701, National Nature Science Foundation of China Grant 91132302, and Strategic Priority Research Program of Chinese Academy of Sciences (B) Grants XDB02010001 and XDB02050001.

\section{References}

Alais, D., Blake, R., \& Lee, S.-H. (1998). Visual features that vary together over time group together over space. Nature Neuroscience, 1 , 160-164. doi:10.1038/414

Beck, D. M., \& Palmer, S. E. (2002). Top-down influences on perceptual grouping. Journal of Experimental Psychology: Human Perception and Performance, 28, 1071-1084. doi:10.1037/0096-1523.28.5. 1071
Blake, R., \& Yang, Y. (1997). Spatial and temporal coherence in perceptual binding. Proceedings of the National Academy of Sciences, 94, 7115-7119. doi:10.1073/pnas.94.13.7115

Brainard, D. H. (1997). The Psychophysics Toolbox. Spatial Vision, 10, 433-436.

Brooks, J. L. (2015). Traditional and new principles of perceptual grouping. In J. Wagemans (Ed.), Oxford Handbook of perceptual organization (pp. 1-31). Oxford: Oxford University Press.

Claessens, P. M. E., \& Wagemans, J. (2005). Perceptual grouping in Gabor lattices: Proximity and alignment. Perception \& Psychophysics, 67, 1446-1459. doi:10.3758/BF03193649

Claessens, P. M. E., \& Wagemans, J. (2008). A Bayesian framework for cue integration in multistable grouping: Proximity, collinearity, and orientation priors in zigzag lattices. Journal of Vision, 8(7), 33.133.23. doi:10.1167/8.7.33

Flanagan, P., Cavanagh, P., \& Favreau, O. E. (1990). Independent orientation-selective mechanisms for the cardinal directions of colour space. Vision Research, 30, 769-778. doi:10.1016/00426989(90)90102-Q

Gibson, J. J., \& Radner, M. (1937). Adaptation, after-effect and constrast in the perception of tilted lines. Journal of Experimental Psychology, 20, 453-467. doi:10.1037/h0059826

Grünbaum, B., \& Shephard, G. C. (1987). Tilings and patterns. New York: WH Freeman.

He, S., \& MacLeod, D. I. A. (2001). Orientation-selective adaptation and tilt after-effect from invisible patterns. Nature, 411, 473-476. doi:10. 1038/35078072

Held, R., \& Shattuck, S. R. (1971). Color- and edge-sensitive channels in the human visual system: Tuning for orientation. Science, 174, 314 316.

Hochberg, J., \& McAlister, E. (1953). A quantitative approach to figural "goodness.". Journal of Experimental Psychology, 46, 361-364. doi:10.1037/h0055809

Kosovicheva, A. A., Maus, G. W., Anstis, S., Cavanagh, P., Tse, P. U., \& Whitney, D. (2012). The motion-induced shift in the perceived location of a grating also shifts its aftereffect. Journal of Vision, 12(8), 1-14. doi:10.1167/12.8.7

Kubovy, M., Holcombe, A. O., \& Wagemans, J. (1998). On the lawfulness of grouping by proximity. Cognitive Psychology, 35, 71-98. doi:10.1006/cogp.1997.0673

Kubovy, M., \& Van den Berg, M. (2008). The whole is equal to the sum of its parts: A probabilistic model of grouping by proximity and similarity in regular patterns. Psychological Review, 115, 131-154. doi:10.1037/0033-295X.115.1.131

Kubovy, M., \& Wagemans, J. (1995). Grouping by proximity and multistability in dot lattices: A quantitative Gestalt theory. Psychological Science, 6, 225-234. doi:10.1111/j.1467-9280.1995. tb00597.x 
Lee, S.-H., \& Blake, R. (1999). Visual form created solely from temporal structure. Science, 284, 1165-1168. doi:10.1126/science.284.5417. 1165

Lovegrove, W. J., \& Over, R. (1973). Colour selectivity in orientation masking and aftereffect. Vision Research, 13, 895-901. doi:10. 1016/0042-6989(73)90069-2

Oyama, T. (1961). Perceptual grouping as a function of proximity. Perceptual and Motor Skills, 13, 305-306. doi:10.2466/pms.1961. 13.3.305

Oyama, T., Simizu, M., \& Tozawa, J. (1999). Effects of similarity on apparent motion and perceptual grouping. Perception, 28, 739748. doi:10.1068/p2799

Palmer, S. E. (1992). Common region: A new principle of perceptual grouping. Cognitive Psychology, 24, 436-447. doi:10.1016/00100285(92)90014-S

Palmer, S. E., \& Beck, D. M. (2007). The repetition discrimination task: An objective method for studying perceptual grouping. Perception \& Psychophysics, 69, 68-78. doi:10.3758/BF03194454

Palmer, S. E., \& Rock, I. (1994). Rethinking perceptual organization: The role of uniform connectedness. Psychonomic Bulletin \& Review, 1, 29-55. doi:10.3758/BF03200760
Pelli, D. G. (1997). The VideoToolbox software for visual psychophysics: Transforming numbers into movies. Spatial Vision, 10, 437-442. doi:10.1163/156856897X00366

Quinlan, P. T., \& Wilton, R. N. (1998). Grouping by proximity or similarity? Competition between the Gestalt principles in vision. Perception, 27, 417-430. doi:10.1068/p270417

Rush, G. P. (1937). Visual grouping in relation to age (Monograph). Archives of Psychology, 31(Whole No. 217).

Wagemans, J., Elder, J. H., Kubovy, M., Palmer, S. E., Peterson, M. A., Singh, M., \& von der Heydt, R. (2012). A century of Gestalt psychology in visual perception: I. Perceptual grouping and figureground organization. Psychological Bulletin, 138, 1172-1217. doi: $10.1037 / \mathrm{a} 0029333$

Wertheimer, M. (1922). Untersuchungen zur Lehre von der Gestalt: I. Prinzipielle Bemerkungen [Investigations in Gestalt science: I. Fundamental remarks]. Psychologische Forschung, 1, 47-58.

Wertheimer, M. (1923). Untersuchungen zur Lehre von der Gestalt: II [Investigations in Gestalt science: II]. Psychologische Forschung, 4, 301-350. doi:10.1007/BF00410640 